\title{
Subject Element Start Date Time
}

National Cancer Institute

\section{Source}

National Cancer Institute. Subject Element Start Date Time. NCI Thesaurus. Code C83411.

The date and time a subject element began. 